\title{
Frequency and determinants of thyroid autoimmunity in Ghanaian type 2 diabetes patients: a case-control study
}

\author{
Osei Sarfo-Kantanka ${ }^{*^{*}}$ (D), Fred Stephen Sarfo ${ }^{1,2}$, Eunice Oparebea Ansah ${ }^{1}$, Ernest Yorke ${ }^{3}$, Josephine Akpalu
} Bernard C. Nkum ${ }^{1,2}$ and Benjamin Eghan ${ }^{1,2}$

\begin{abstract}
Background: The link between type 1 diabetes and thyroid autoimmunity is well described. The same cannot be said for type 2 diabetes where results have been mixed so far. We investigated the prevalence and determinants of thyroid autoimmunity among Ghanaian type 2 diabetes patients.

Methods: This was a case-control study involving 302 type 2 diabetes patients and 310 non - diabetic controls aged 40-80 years. Anthropometric and blood pressure measurements were obtained. Fasting samples were analyzed for glucose, thyroid function, and antibodies to thyroglobulin and thyroid peroxidase.

Results: The prevalence of thyroid autoimmunity was significantly higher among T2DM subjects (12.2\% vs. 3.9\%, $p=0$. 0004). Among T2DM subjects, 44 (14.7\%) tested positive for TPOAb, 5 (1.7\%) tested positive for TGAb and 15 (5.0\%) tested positive for both autoantibodies. Females T2DM subjects showed a 3-fold increased risk of thyroid autoimmunity compared to males (OR:3.16, $p=0.004$ ), T2DM subjects with hyperthyroidism had a $41 \%$ increased risk of thyroid autoimmunity (OR: 1.41, $p<0.001$ ), sub-clinical hyperthyroidism increased the risk of thyroid autoimmunity by 2 fold, (OR:2.19, $p<0.001)$, subclinical hypothyroidism increased the risk of autoimmunity by 4 -fold, (OR:3.57 95\% $p<0$. 0001), and hypothyroidism was associated with a 61\% increased risk of thyroid autoimmunity (OR: 1.61,1.35-2.23). Dyslipidaemia was associated with a $44 \%$ increased risk of thyroid autoimmunity (OR: 1.44, $p=0.01)$ and a percentage increase in $\mathrm{HbA1c}$ was associated with $46 \%$ increased risk of thyroid autoimmunity (OR:1.46, $p<0.0001)$.

Conclusion: We observed a high prevalence of thyroid autoimmunity in Ghanaian T2DM subjects compared to the general population. Thyroid autoimmunity in T2DM subjects was significantly associated with female gender, thyroid dysfunction, dyslipidaemia and poor glycemic control.
\end{abstract}

Keywords: Thyroid autoimmunity, Type 2 diabetes mellitus, Associated factors

\section{Background}

Diabetes and thyroid disorders represent the two commonest endocrinological conditions seen in adult medical practice $[1,2]$. The concurrence of the two conditions in the same individual can prove inimical to achieving good glycemic control and further multiply the cardiovascular risk associated with diabetes. $[1,2]$ Studies worldwide have shown a higher prevalence of thyroid dysfunction in type 2 diabetes (T2DM) patients and vice-versa [2-8]. The

\footnotetext{
*Correspondence: osarfokantanka21@gmail.com

'Directorate of Medicine, Komfo Anokye Teaching Hospital, Endocrine and Diabetes Unit, P.O. Box 1934, Kumasi, Ghana

Full list of author information is available at the end of the article
}

spectrum of thyroid disorders (like diabetes) is wide; and it is continuously experiencing a change in epidemiology, usually determined by iodine levels seen in the population in focus $[9,10]$. In arears of the world where intake of iodine, a major component of thyroid hormones, is sufficient, autoimmune disorders represent the commonest cause of thyroid pathology [11]. In contrast, there is widespread dietary iodine deficiency in Africa, which underlines most of the clinical and pathological presentations of thyroid disease [12]. Recently, with the remarkable improvement in iodine nutrition through widespread salt iodination on the continent, there appears to be a shift in thyroid epidemiology towards autoimmunity [13]. Thyroid 
autoimmunity which comprises a number of distinct but pathogenically related immune-mediated destructive disorders of the thyroid gland is often characterized by the presence of autoantibodies directed mostly against thyroid peroxidase (TPOAb) and thyroglobulin(TGAb) [14]. Type 1 diabetes has an established association with autoimmune thyroid disorders through a common genetic inheritance $[15,16]$. Studies to investigate a link between thyroid autoimmunity and T2DM have produced mixed results so far, mostly beset by differing methodologies, iodine statuses and sensitivities of immunological tests employed in determining thyroid autoantibodies [17-21]. Whiles Akbar et al. [4] obtained a significantly higher prevalence of thyroid autoimmunity in the study of Saudi T2DM subjects, Afkhami- Ardekani et al. [22] study of Iranian T2DM subjects did not yield any significant difference between the two groups. Among Africans, there exist a gaping hole of literature documenting thyroid autoimmunity both among the general population and T2DM patients. Cardoso et al. in one of the few studies on the continent to date, compared type 1 diabetes patients and controls with T2DM subjects for thyroid autoantibodies and obtained a predictably low autoantibody level of $1.7 \%$ among T2DM subjects [23]. As far as we are aware, no recent published studies were cited on the topic on the continent to reflect the changing epidemiology of thyroid diseases among Africans toward increased autoimmunity. The aim of this study was therefore to determine the prevalence and the associated factors of thyroid autoimmunity in Ghanaian T2DM patients.

\section{Methods}

This was a case-control study in which cases were consecutive patients with established T2DM defined by the WHO criteria [18], self-reported diagnosis of diabetes and/or treatment with antidiabetic medications (among patients who were insulin non-requiring in the first year after diagnosis for glycemic control). Cases were recruited from the outpatient diabetes clinic of Komfo Anokye Teaching Hospital (KATH), the second largest tertiary referral hospital in Ghana from April 2014 to April 2015. Community in-dwelling age and sex matched adults from the same region were recruited to serve as controls after normoglycemia was documented by both fasting plasma glucose (FPG) and glycated hemoglobin (HbA1c). Using a structured validated questionnaire and a review of medical records we obtained the sociodemographic and clinical information of all participants. Because of their confounding effects on thyroid function, we excluded pregnant women, patients on amiodarone, lithium and long-term corticosteroids as well as those with an acute illness and history of hospitalization less than 6 months from the day of recruitment.

\section{Ethical approval and consent to participate}

The study was approved by the Committee for Human Research Publications and Ethics at the School of Medical Sciences, Kwame Nkrumah University of Science and Technology and the Komfo Anokye Teaching Hospital, Kumasi. All participants gave an informed consent with those unable to understand or sign the informed consent excluded.

\section{Study measurements \\ Physical measurements}

Body weight and height were taken in duplicates using a combined manual scale and stadiometer (Asimed MB 211 T plus Asparatos Y Sistemas de Medida,). Body mass Index (BMI) was calculated as weight in kilogram divided by the square of height in meters $\left(\mathrm{kg} / \mathrm{m}^{2}\right)$. Overweight/general obesity was defined as BMI $\geq 25 \mathrm{~kg} / \mathrm{m}^{2}$ [17].

\section{Waist circumference measurement}

Duplicate waist circumference (WC) measurements were taken and the average recorded for both group of participants, WC measurements $>80 \mathrm{~cm}$ and $94 \mathrm{~cm}$ were recorded as central obesity for females and males respectively [16].

\section{Blood pressure measurement}

Duplicate blood pressure recordings were taken with the participant in a seated upright position using a standard mercury sphygmomanometer after at least $15 \mathrm{~min}$ of rest. Hypertension was defined as mean blood pressure $\geq$ $140 / 90 \mathrm{~mm} \mathrm{Hg}$ and/ or documented antihypertensive therapy [19].

Smokers were identified by self-report as those who had smoked at least 10 sticks of cigarette per day for 6 months or more or those who smoked daily for 1 year or more regardless of the number of cigarettes smoked per day [20]. Positive alcohol intake status was identified when greater than 14 units of alcohol was consumed per week in the case of a female and 21 units per week in case of a male [21].

\section{Laboratory measurements}

Approximately ten milliliters $(10 \mathrm{mls})$ of fasting venous samples were collected from each participant into vacutainer tubes (Becton Dickinson, Rutherford, and N.J) and Sequestrene bottles. Samples were manually processed and cryopreserved before transporting to laboratory for analysis. Fasting plasma glucose (FPG), thyroid profile: free thyroxine (FT4), free triiodothyronine (FT3), thyroid stimulating hormone (TSH), total cholesterol (TC), low-density lipoprotein cholesterol (LDL-C), high-density lipoprotein cholesterol (HDLC), triglycerides (TG)], urea, creatinine, TGAb and 
TPOAb were assayed by Chemoimmunoluminiscence method (Roche Diagnostics, Cobas e411 automated immunoassay analyzer, Indianapolis, USA) following the manufacturer's instructions. Glycated hemoglobin (HbA1c) measurements were performed by standardized high performance liquid chromatography assay using Bio-Rad Variant II hemoglobin testing autoanalyzer. The reference range, intra-assay and interassay coefficients of variation for thyroid hormones and antibodies were as follows:

(TSH: $0.25-5.0 \mathrm{IU} / \mathrm{ml},<2.1 \%$ and $<2.4 \%$, FT3: $3.7-$ $10.4 \mathrm{pmol} / \mathrm{l}, 5.8 \%$ and $6.9 \%$ for FT3, FT4: $7.5-21.1 \mathrm{pmol} / \mathrm{l}$, $2.8 \%$ and $2.4 \%, \mathrm{TPOAb}>5.6 \mathrm{U} / \mathrm{L}, 2.1 \%$ and $6.1 \%$, $\mathrm{TGAb}>4.1 \mathrm{U} / \mathrm{L}, 1.9 \%$ and $5.6 \%$ ).

Thyroid function was classified as: Euthyroidism when FT4, FT3 and TSH were within the normal range, hypothyroidism when TSH level was greater than the upper limit of the reference range and FT4/ or FT3 is lower than the lower limit of their reference ranges, Subclinical hypothyroidism- when TSH is greater than the upper limit of the reference range and FT4 and FT3 are within the normal range. Hyperthyroidism- when TSH level is lower than the upper limit of the reference range and FT4/or FT3 is greater than the upper limit of their reference ranges, subclinical hyperthyroidismwhen TSH level is lower than the lower limit of the reference range and $\mathrm{FT}_{3}$ and $\mathrm{FT}_{4}$ are within the normal range. Thyroid autoimmunity was defined as positive TPOAb and/or TGAb.

Dyslipidemia was defined as TG level $\geq 3.0 \mathrm{mmol} / \mathrm{L}$ and HDL cholesterol level $(<1.0 \mathrm{mmol} / \mathrm{L})$ regardless of patient's gender [24].

\section{Statistical analysis}

Data was analyzed using Graph Pad Prism 7 software for Mac OS X. Continuous and dichotomous variables were presented as mean (standard deviation) and $\mathrm{n}$ (\%) respectively. Data normality assumption was performed by visual inspection of distribution as well as D Agostino and Pearson Omnibus normality test. Statistical difference between means, medians and proportions were assessed using student t-tests, Mann-Whitney $U$ tests and chi-square test respectively. To adjust for the effects of confounders, logistic regression models was carried out to identify independent predictors of thyroid autoimmunity. A significant level of $P<0.05$ was used for the analysis.

\section{Results and discussion}

\section{Baseline characteristics of T2DM subjects and controls}

The overall study population was 612 (comprising 302 T2DM subjects and 310 controls). Table 1 describes the baseline characteristics of T2DM subjects and controls. There was no difference in age $(57.6 \pm 9.4$ vs. $57.2 \pm 9.5$, $p=0.65)$ and percentage of females (58.9 vs 58.4, $p=$ $0.95)$ between the 2 groups of participants. Type 2 diabetes subjects had significantly higher mean systolic blood pressure ( $148.2 \pm 20.5$ vs. $130.4 \pm 22.7, p<0.0001)$, mean diastolic blood pressure (82.6 \pm 12.6 vs. $77.6 \pm 12.7$, $p<0.0001)$, median BMI [27.9 (21.5-31.5) vs. 27.0 (24$30.3, p=0.03)$ ], waist circumference [98 $(90-106)$ vs. 94 $(84-100.8) p<0.0001]$ and proportion with dyslipidemia (53\% vs. $11 \%, p<0.0001)$ compared to controls. The proportion of participants that smoked or drank alcohol was not significantly different between the two groups.

\section{Prevalence of thyroid dysfunction and autoimmunity between the two groups}

As shown in Table 1, the prevalence of thyroid dysfunction between the two groups was not significant (18.5 vs. $12.9, p=0.06)$. The prevalence of thyroid autoimmunity among T2DM participants was about 5fold higher than in controls $(21 \%$ vs $4 \%, p<0.0001)$. Of the 302 T2DM subjects, $14.7 \%(n=44)$ tested positive for TPOAb, 1.7\% $(n=5)$ for TGAb and 5\% $(n=$ $15)$ for both antibodies. For controls $2 \%(n=6), 1 \%(n$ $=3)$ and $1 \%(n=3)$ tested positive for TPOAb, TGAb and both antibodies respectively. Significant difference existed in the two groups in the prevalence of TPOAb (14.7\% vs $1.9, p<0.0001)$, TPOAb and TGAb $(5.0 \%$ vs $0.9 \%, p=0.004)$. The difference in prevalence of TGAb between the two groups was not significant $(1.7 \%$ vs $0.9 \%, p=0.95)$.

Figure 1 shows that the median concentration of TPOAb was significantly higher in T2DM patients compared to controls [4.5(2.9-6.3) vs 2.0(1.2-3.6), $p<$ $0.0001]$. The median concentration of TGAb was not significantly different between the groups [2.4 (1.8-2.8) vs 2.3 (1.6-2.8), $p=0.95$ ].

\section{Frequency of thyroid thyroid dysfunction among autoimmunity positive participants}

Figure 2 shows the frequency of thyroid dysfunction among autoimmune positive participants. Of the 44 T2DM subjects who tested positive for TPOAb, 68\% $(n=30)$ had thyroid dysfunction, 20\% $(n=1)$ of the 5 patients who tested positive for TGAb had thyroid dysfunction whiles $93 \%(n=14)$ of the 15 patients with both antibodies had thyroid dysfunction. Among controls, $17 \%(n=1)$ of TPOAb positive had thyroid dysfunction, $20 \%(n=1)$ of those with TGAb and TPOAb had thyroid dysfunction. The presence of TPOAb and both antibodies was significantly associated with thyroid dysfunction in T2DM subjects compared to controls. The difference in the proportion of TGAb positive participants with thyroid dysfunction was not significant between the two groups. 
Table 1 Demographic and Clinical Characteristics of Study participants

\begin{tabular}{|c|c|c|c|}
\hline Variable & T2DM subjects & Controls & $P$ - Value \\
\hline Number & 302 & 310 & \\
\hline Age, years (mean $\pm S D)$ & $57.6 \pm 9.4$ & $57.2 \pm 9.5$ & 0.65 \\
\hline Hypertension, n(\%) & $213(70.5)$ & $75(24.5)$ & $<0.0001$ \\
\hline Female gender, n (\%) & $178(58.9)$ & $181(58.4)$ & 0.95 \\
\hline $\mathrm{SBP}, \mathrm{mmHg}($ mean $\pm \mathrm{SD})$ & $148.2 \pm 20.5$ & $130.4 \pm 22.7$ & $<0.0001$ \\
\hline $\mathrm{DBP}, \mathrm{mmHg}($ Mean $\pm \mathrm{SD})$ & $82.6 \pm 12.6$ & $77.6 \pm 12.7$ & $<0.0001$ \\
\hline Dyslipidemia, n (\%) & $159(52.6)$ & $24(11.4)$ & $<0.0001$ \\
\hline Alcohol, n (\%) & $87(28.8)$ & $120(29.0)$ & 0.96 \\
\hline Smoking, n (\%) & $22(7.3)$ & $15(4.8)$ & 0.24 \\
\hline Body mass index, kg/m², median (IQR) & $27.9(21.5-31.5)$ & $27.0(24-30.3)$ & 0.03 \\
\hline Waist Circumference, cm, median (IQR) & $98(90-106)$ & $94(84-100.8)$ & $<0.0001$ \\
\hline Central obesity, n (\%) & $210(69.5)$ & $121(39.0)$ & $<0.0001$ \\
\hline $\mathrm{HbA} 1 \mathrm{c}, \%$, median (IQR) & $8.2(6.9-9.6)$ & $5.2(5.0-5.4)$ & $<0.0001$ \\
\hline Fasting blood glucose, $\mathrm{mmol} / \mathrm{L}$, (Mean $\pm \mathrm{SD})$ & $10.4 \pm 5.4$ & $5.3 \pm 1.4$ & $<0.0001$ \\
\hline Creatinine, $\mu \mathrm{mol} / \mathrm{L}$, median (IQR) & $84(62-106.8)$ & $77(63-99)$ & 0.21 \\
\hline Thyroid dysfunction, n (\%) & $56(18.5)$ & $40(12.9)$ & 0.06 \\
\hline Thyroid autoimmunity, n (\%) & $64(21.2)$ & $12(3.9)$ & $<0.0001$ \\
\hline Positive TPOAb, n (\%) & $44(14.7)$ & $6(1.9)$ & $<0.0001$ \\
\hline Positive TGAb, n (\%) & $5(1.7)$ & $3(0.9)$ & 0.99 \\
\hline Positive TPOAb + TGAb n (\%) & $15(5.0)$ & $3(0.9)$ & 0.004 \\
\hline
\end{tabular}

\section{Clinical and laboratory characteristics of T2DM subjects according to autoantibody status}

As shown in Table 2, T2DM subjects with thyroid autoimmunity had significantly higher FPG [9.7 (8.7-11.2 VS. 7.6 (6.7-9.4), $p<0.0001]$, HbA1c [8.2 (6.8-9.8) vs. $6.1(5.2-9.6) p<0.0001]$, TC $[6.03(4.7-7.06)$ vs. 4.9 (3.9-5.7), $p<0.0001]$, LDL-C [3.87 (2.83-5.08) vs. 2.90 $(2.0-3.60), \quad p<0.0001]$ and TSH $[3.4 \quad(0.1-8.1)$ vs $1.1(0.8-1.8) p<0.04]$ compared to T2DM subjects without thyroid autoimmunity. There was no significant

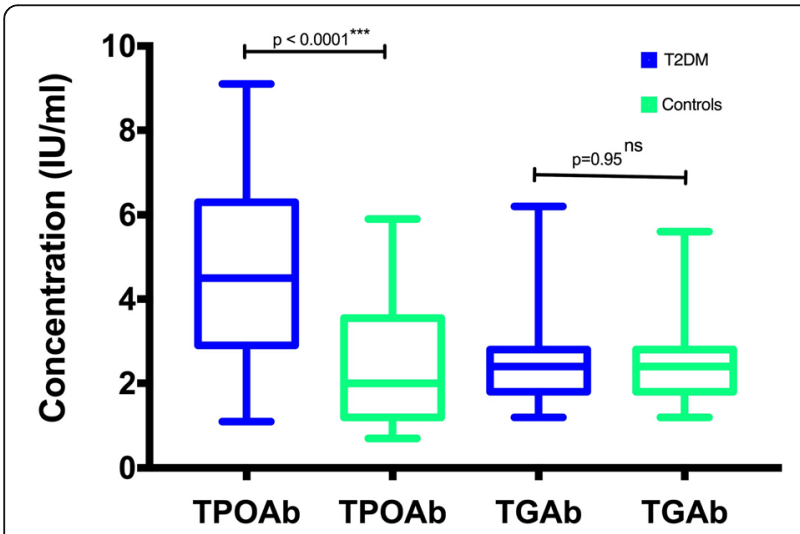

Fig. 1 Mean concentration of thyroid autoantibodies in study participants difference between the two groups in terms of age, duration of diabetes, blood pressure and renal function.

\section{Associations of thyroid autoimmunity in T2DM subjects}

The results of multiple logistic regression analysis are shown in Table 3 were as follows; after adjusting for BMI, T2DM subjects with thyroid autoimmunity had a 3-fold increased risk of being females, (OR: 3.16 95\% CI: $1.46-6.87, p<0.0001)$, a percentage increase in $\mathrm{HbA} 1 \mathrm{c}$ increased the odds of thyroid autoimmunity by $46 \%$ (OR: 1.46 95\% CI 1.23-1.73) and a mmol increase in TC increased the odds of thyroid autoimmunity by $44 \%$. The odds of thyroid dysfunction

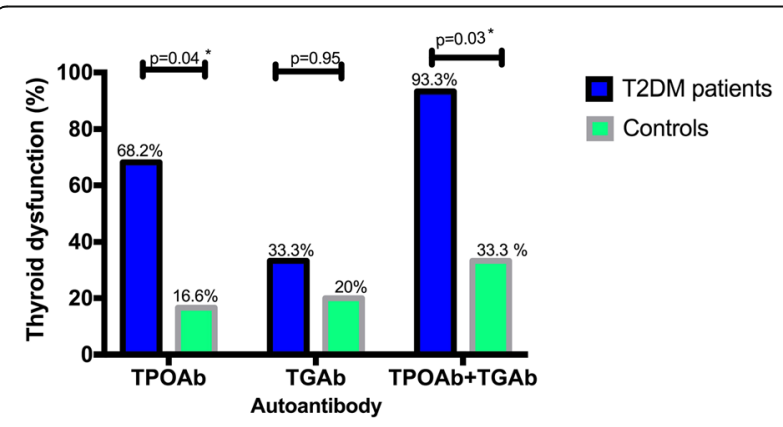

Fig. 2 Thyroid dysfunction in autoantibody positive T2DM subjects and Controls 
Table 2 Characteristics of T2DM subjects according to thyroid autoimmunity status

\begin{tabular}{|c|c|c|c|}
\hline Variable & Positive Thyroid autoimmunity & Negative thyroid autoimmunity & $P$ \\
\hline Number (\%) & $64(21.2)$ & $239(78.8)$ & $<0.0001$ \\
\hline Female Gender, n (\%) & $58(70)$ & $138(57.7)$ & 0.04 \\
\hline Age, years, (mean $\pm S D)$ & $57.5 \pm 9.0$ & $57.4 \pm 9.5$ & 0.93 \\
\hline Duration of T2DM, median (IQR) & $5(3-10)$ & $5(2-10)$ & 0.49 \\
\hline Systolic blood pressure, median (IQR) & $140(130-160)$ & $139(130-150)$ & 0.33 \\
\hline Diastolic blood pressure, median (IQR) & $85.5(70-90)$ & $80(72-90)$ & 0.31 \\
\hline Smoking, n (\%) & $7(13.0)$ & $15(6.3)$ & 0.27 \\
\hline Alcohol, n (\%) & $31(48.4)$ & $86(36.0)$ & 0.08 \\
\hline $\mathrm{BMI}, \mathrm{Kg} / \mathrm{m}^{2}$, median (IQR) & $24.7(24.8-30.4)$ & $26.9(24.5-32.1)$ & 0.79 \\
\hline Waist circumference, cm, median (IQR) & $99(88-109)$ & $95(86-102)$ & 0.04 \\
\hline Fasting blood glucose, mmol/L, median (IQR) & $9.7(8.7-11.2)$ & $7.6(6.7-9.4)$ & $<0.0001$ \\
\hline $\mathrm{HbA} 1 \mathrm{c} \%$ median IQR & $8.2(6.8-9.8)$ & $6.1(5.2-9.6)$ & $<0.001$ \\
\hline TC median IQR & $6.03(4.7-7.06)$ & $4.9(3.9-5.7)$ & $<0.001$ \\
\hline LDL-C, mmol/L median IQR & $3.87(2.83-5.08)$ & $2.90(2.0-3.60)$ & $<0.0001$ \\
\hline HDL-C, mmol/L median (IQR) & $1.22(1.0-1.45)$ & $1.18(1.0-1.5)$ & 0.41 \\
\hline Triglycerides, mmol/L, median (IQR) & $1.25(0.99-1.67)$ & $1.19(0.9-1.60)$ & 0.18 \\
\hline Creatinine, mmol/L, median (IQR) & $84(62-106)$ & 77 (63-99) & 0.21 \\
\hline $\mathrm{TSH}, \mathrm{pmol} / \mathrm{L}$, median (IQR) & $3.4(0.1-8.1)$ & $1.1(0.8-1.8)$ & $<0.04$ \\
\hline
\end{tabular}

were increased in T2DM subjects' with thyroid autoimmunity with a 2-fold increased odds of subclinical hyperthyroidism; (OR: $2.195 \%$ CI: 1.7-2.6, $p<$ $0.0001), 1.41 \times$ increased odds of clinical hyperthyroidism (OR: 1.41: 95\% CI: 1.2-1.98, $p<0.0001$ ), $3.8 \times$ increased odds of subclinical hypothyroidism (OR: 3.8 95\% CI: $2.7-3.8 p<0.0001)$ and $61 \%$ increased odds of clinical hypothyroidism (OR: 1.61 95\% CI :1.35$2.23, p<0.0001)$.

\section{Discussion}

There are limited studies comparing autoantibody prevalence between T2DM subjects and controls worldwide. Our study has shown a higher prevalence of thyroid

Table 3 Multiple Logistic Regression Analysis for determinants of autoimmunity in T2DM patients

\begin{tabular}{|c|c|c|c|c|}
\hline Variable & $\begin{array}{l}\text { Unadjusted Odds ratio } \\
95 \% \mathrm{Cl}\end{array}$ & $p$-value & $\begin{array}{l}\text { Adjusted Odds ratio } \\
95 \% \mathrm{Cl}\end{array}$ & $p$-value \\
\hline \multicolumn{5}{|l|}{ Age } \\
\hline For each 10 years older & $1.00(0.88-1.15)$ & 0.96 & - & - \\
\hline \multicolumn{5}{|l|}{ Gender } \\
\hline Female & $4.45(2.17-9.15)$ & $<0.0001$ & $3.16(1.46-6.87)$ & 0.004 \\
\hline Male & 1.00 & & & \\
\hline \multicolumn{5}{|l|}{ Thyroid function } \\
\hline Euthyroidism & 1.00 & - & & \\
\hline Clinical hyperthyroidism & $1.98(1.04-3.04)$ & $<0.0001$ & $1.41(1.20-1.98)$ & $<0.0001$ \\
\hline Clinical hypothyroidism & $2.21(2.11-3.41)$ & $<0.0001$ & $1.61(1.35-2.23)$ & $<0.0001$ \\
\hline Subclinical hyperthyroidism & $2.5(2.19-2.90)$ & $<0.0001$ & $2.19(1.70-2.58)$ & $<0.0001$ \\
\hline Subclinical hypothyroidism & $5.0(4.60-5.50)$ & $<0.0001$ & $3.57(2.74-3.82)$ & $<0.0001$ \\
\hline \multicolumn{5}{|l|}{ Glycaemic state } \\
\hline For every $1 \%$ increase in $\mathrm{HbA} 1 \mathrm{c}$ & $1.83(1.53-2.19)$ & $<0.0001$ & $1.46(1.23-1.73)$ & $<0.0001$ \\
\hline \multicolumn{5}{|l|}{ Cholesterol status } \\
\hline For $1 \mathrm{mmol}$ increase in total Cholesterol & $1.55(1.28-1.87)$ & $<0.0001$ & $1.44(1.17-1.77)$ & 0.01 \\
\hline
\end{tabular}


autoimmunity in Ghanaian T2DM subjects compared to controls with one in five T2DM subjects testing positive for thyroid autoimmunity compared to one in twenty seen among the controls. This finding is consistent with those of Akbar [4], Yasmin [25] and Konstantinos [26] who recorded significantly higher prevalence of thyroid autoimmunity in T2DM subjects compared to controls, with prevalence ranging between $10 \%$ and $43 \%$ among T2DM subjects. On the contrary, Cardoso et al. [4] and Afkhami- Ardekani et al. [22] recorded no significant difference between the 2 groups. The discrepancy in the results of studies investigating the prevalence of thyroid autoimmunity in T2DM subjects may be as a result of different methodologies employed in the determination of autoantibodies. It has been shown that the prevalence of these autoantibodies increases as the sensitivity of the assay method increase. This may have accounted for the highly significant increase in prevalence of autoantibody prevalence seen in our study. Cardoso employed manual ELISA methods whiles we used a more sensitive 2- site Chemiluminescent automated method in our determination [27]. Additionally, differences in case groups, especially in terms of differing ages, race and ethnicity, varying sample sizes, gender composition, geographic area, duration of diabetes of subjects in the individual studies may have accounted for the difference in results obtained. Although not tested in our study, it has been shown that T2DM subjects have reduced levels of Vitamin D $[28,29]$, a situation that can trigger autoimmunity and serve as a link between T2DM and thyroid autoimmunity as seen in our study.

Autoimmune disorders generally, including thyroid autoimmunity, are commonly associated with female gender compared to males due to the role of estrogen as an immunomodulator [29]. Additionally, there is an increased susceptibility of females to antibody formation in response to stress due to an increased $\mathrm{T}$ helper (Th) 2- predominant immune response compared to male where cytotoxic response is elicited from $\mathrm{T}$ helper $(\mathrm{Th})$ 1 response [30]. Similarly, our study found that females T2DM subjects had a 3-fold increase risk of thyroid autoimmunity compared to males. Our study had a significantly higher representation of females though.

There was varying concentration of thyroid antibodies among T2DM subjects and controls with the median level of TPOAb significantly higher among T2DM subject compared to controls. With respect to TGAb there was no significant difference between the two groups. The higher levels of TPOAb may be due to the increased stimulation of thyroid autoantibody collaborating well with increased lymphocytic infiltration of the thyroid gland [31].

The presence of thyroid autoimmunity was significantly associated with subclinical thyroid disease with almost 3 fold increased risk of subclinical thyroid disease. Clinical thyroid disease including hyperthyroidism and hypothyroidism were also increased by about $1.5 \times$ fold in the presence of thyroid autoimmunity. This finding suggests that the presence of thyroid antibodies may serve as an indicator of both overt and subclinical thyroid dysfunction [32, 33]. Majority of those with subclinical disease may in the presence of thyroid autoantibodies expected to progress to overt thyroid disease as seen in participants of the Freemantle study [34].

In T2DM subjects with thyroid dysfunction, there is an increase insulin resistance usually manifesting as worsened lipid levels and poor glycemic controls [35, 36]. This is seen in our study where patient with thyroid autoimmunity most of which was associated with thyroid dysfunction had an almost 2-fold higher levels of glycated hemoglobin and dyslipidaemia. Additionally, it has been shown that thyroid autoimmunity correlate well with autoimmune destruction of beta- cells (though not a significant pathophysiology in T2DM), and this can lead to worsening of glycaemic control as seen in our study [37].

A major limitation of this study was our inability to test for Thyroid Stimulating Immunoglobulins which may indicated the cause of hyperthyroidism in some of the cases. Additionally, our inability to test for Glutamic Acid Decarboxylase Autoantibody type 65 (GAD 65) especially in T2DM subjects in the early forties meant we may have enrolled patients with Latent Autoimmune Diabetes of Adults in the study. Also to be noted is our inability to use oral glucose tolerance test in ruling out diabetes in our controls. With this, a marginal misclassification of patients with glucose intolerance may have been included as controls. Considering the observational nature of this evidence and, thus, the inappropriateness for causality inference, we advise caution in the interpretation of these findings. Especially in extrapolating these findings to different populations with different baseline characteristics. However, the impact of these limitations on our study findings is probably minimal, since the discrimination between the T2DM subjects and the control group was based on two glycemic indices (fasting glucose and HbA1c measurements), which secured a clear distinction between groups.

The strength of this study compared to a comparable study among West Africans is the increased sample size of 310 T2DM subjects compared to 60 patients in the first study. Future studies should be designed to study the influence of other factors including Vitamin D status on thyroid autoimmunity in T2DM subjects.

\section{Conclusion}

The results of the present study indicate that the frequency of thyroid autoimmunity is significantly higher 
in Ghanaian T2DM patients, with its presence significantly associated with thyroid dysfunction, female gender, hypercholesterolemia and hyperglycemia. Therefore, it is necessary to screen type 2 diabetes patients especially females with thyroid dysfunction for thyroid autoimmunity.

\section{Additional file}

Additional file 1: Excel File with Participant Information. (XLSX 125 kb)

\section{Abbreviations}

BMI: Body mass index; FT3: Free triiodothyronine; FT4: Free thyroxine; HDLC: High-density lipoprotein cholesterol; LDL-C: Low-density lipoprotein cholesterol; T2DM: Type 2 diabetes mellitus; TC: Total cholesterol; TG: Triglycerides; TGAb: Thyroglobulin autoantibody; TPOAb: Thyroid peroxidase autoantibody; WC: Waist circumference

\section{Acknowledgments}

The authors acknowledge the immense role played by Nurses and laboratory scientists at the Diabetes Clinic, KATH. We also appreciate the contribution of staff at the office of the Department of medicine, KATH who helped in typing the manuscript. We are eternally grateful to all participants for participating in the study.

\section{Funding}

This study was funded by the lead author.

\section{Availability of data and material}

The data upon which this study was reported has been attached as Additional file 1.

\section{Authors' contributions}

OSK conceived the study, participated in its design and drafted the manuscript. FSS contributed in study design and coordinated data collection and helped with statistical analysis. EOA contributed in conducting the field activities. EY contributed in the study design and manuscript development. JA participated in the design of the study and performed the statistical analysis JCM participated in the manuscript development. BE and BN helped organized and put the manuscript together. All authors read and approved the final version of the manuscript.

\section{Competing interests}

There is no competing interest involving any of the authors of this study.

\section{Consent for publication}

All participants in this study consented to information obtained from them for this study to be published.

\section{Ethics approval and consent to participate}

The study was approved by the Committee for Human Research Publications and Ethics at the School of Medical Sciences, Kwame Nkrumah University of Science and Technology and the Komfo Anokye Teaching Hospital, Kumasi. All participants gave an informed consent with those unable to understand or sign the informed consent excluded.

\section{Author details}

'Directorate of Medicine, Komfo Anokye Teaching Hospital, Endocrine and Diabetes Unit, P.O. Box 1934, Kumasi, Ghana. ²Department of Medicine, School of Medical Sciences, Kwame Nkrumah University of Science and Technology, Kumasi, Ghana. ${ }^{3}$ Department of Medicine, University of Ghana School of Medicine and Dentistry, Accra, Ghana.

Received: 20 September 2016 Accepted: 29 December 2016 Published online: 17 January 2017

\section{References}

1. Sathish R, Mohan V. Diabetes and Thyroid diseases- a review. Int J Diab Dev Ctries. 2003;23(4):120-3.

2. Al-Wazzan HT, Daban AH, Askar RA. El- Shazly MK. Prevalence and associated factors of thyroid dysfunction among type 2 diabetic patients in Kuwait. Alex Bull. 2010:46(2):141-8.

3. Papazafiropoulou A, Sotiropoulos A, Kokolaki A, Kodora M, Stamatai P, Stavros P. Prevalence of thyroid dysfunction amongst Greek Type 2 Diabetes Mellitus patients attending an outpatient Clinic. J Clin Med Res. 2010;2(2):75-8.

4. Akbar DH, Ahmed MM, Al-Mughales J. Thyroid dysfunction and thyroid autoimmunity in Saudi type 2 diabetics. ACTA Diabetologia. 2006;43(1):14-8.

5. Ghazali SM, Abbiyesuku FM. Thyroid dysfunction in type 2 diabetics seen at the University College Hospital, Ibadan, Nigeria. Niger J Physiol Sci. 2010;25(2):173-9.

6. Radaideh AR, Nusier MK, Amari FL, Bateiha AE, El-Khateeb MS, Naser AS, Ajlouni KM. Thyroid dysfunction in patients with type 2 diabetes mellitus in Jordan. Saudi Med J. 2004;25(8):1046-50.

7. Perros $P, M c C$ rimmon RJ, Shaw G, Frier BM. Frequency of thyroid dysfunction in diabetic patients: value of annual screening. Diabet Med. 1995:12:622-7.

8. Freely J, Isles TE. Screening for thyroid dysfunction in diabetics. Br Med J. 1979:1(6179):1678.

9. Tunbridge WMG, Everd DC, Hall R, Appleton D, Brewis M, Clark F. The spectrum of thyroid disease in the community: The Wickham survey. Clin Endocrinol. 1997;19:481-93.

10. Weetman AP. Diseases associated with thyroid autoimmunity: an explanation for the expanding spectrum. Clin Endocrinol(oxf). 2011;74:411-8.

11. Kahaly GJ, Dienes HP, Beyer J, Hommel G. lodide induces thyroid autoimmunity patients with endemic goiter: A randomized, double-blind, placebo-controlled trial. Eur J Endocrinol. 1998;139:290-70.

12. Hetzel BS. lodine deficiency disorders (IDD) and their eradication. Lancet. 1983:2:1126-9.

13. World Health Organization. Iodine Status Worldwide: WHO Global Database on lodine Deficiency. Geneva: WHO; 2004. p. 1-58. http://www.who.int/ nutrition/publications/micronutrients/FNBvol29N3sep08.pdf?ua=1.

14. Donckier JE. Endocrine diseases and Diabetes. In: Pickup JC, Williams G, editors. Textbook of Diabetes mellitus. Chichester: Black Well Publishing Company; 2003. p. 27.1-27.25.

15. Radetti G, Paganini C, Gentili L, Bernasconi S, Betterle C, Borkenstein M, Cvijovic K, Kadrnka-Lovrencic M, Krzisnik C, Battelino T, et al. Frequency of Hashimoto's thyroiditis in children with type 1 diabetes mellitus. Acta Diabetol. 1995;32:121-4.

16. Umpierrez GE, Latif KA, Murphy MB, Lambeth HC, Stentz F, Bush A, Kitabchi AE. Thyroid dysfunction in patients with type 1 diabetes: a longitudinal study. Diabetes Care. 2003;26(4):1181-5.

17. World Health Organisation. Obesity: Prevention and managing the global epidemic. Report of a WHO consultation. Geneva: WHO Technical Report Series 894: 2000

18. World Health Organisation. Definition, diagnosis and classification of diabetes mellitus and its complications: Report of a WHO Consultation. Part 1. Diagnosis and classification of diabetes mellitus. 2007. Available from www.who.int/diabetes/publications/en/. Accessed 18 June 2015.

19. Chalmers J, McMahon S, Mancia G, Whitwort J, Beilin L, Hansson L, et al. 1999 World Health Organisation-International Society of Hypertension Guidelines for treatment of hypertension. Guideline subcommittee of the World Health Organisation. Clin Exp Hypertens. 1999:21(5-6):1009-60.

20. Wannamethee SG, Shaper AG, Perry IJ. British Regional Heart Study: Smoking as a modifiable risk factor for type 2 diabetes in middle-aged men. Diabetes Care. 2001;204(9):1590-5.

21. Shaper AG, Wanathee $G$, Walker M. Alcohol and Mortality: explaining the $U$ shaped Curve. Lancet. 1988;2:1268-73.

22. Afkhami-Ardekani M, Rashidi M, Shojaoddeny- Ardekani A. Evaluation of thyroid autoantibody in type 2 diabetes. IJDO. 2009;1(1):1-4.

23. Cardoso C, Ohwovoriole AE, Kuku SF. A study of thyroid function and prevalence of thyroid autoantibodies in an African diabetic population. J Diabetes Complications. 1995;9(1):37-41.

24. Jellinger PS, Smith DA, Mehta AE, Ganda O, Handelsman Y, Rodbard HW, et al. American association of clinical endocrinologists' guidelines for management of Dyslipidemia and prevention of atherosclerosis. Endocr Pract. 2012;18 Suppl 1:1-78.

25. Yasmin T, Ghafoor F, Malik T. S RN, Khan AU. The pattern of thyroid autoimmunity in type 1 and type 2 diabetics. J Coll Physicians Surg Pak. 2006;16(12):751-4 
26. Konstantinos, Xanthippi T, Evangelos P, Triantafyllos D, Anna G, Pantelis Z et al. Thyroid Autoimmunity in the Context of Type 2 Diabetes Mellitus: Implications for Vitamin D. Int Journal of Endocrinology 2015; ol. 2015, Article ID 710363, 2015. doi:10.1155/2015/710363.

27. Okosieme OE, Taylor RC, Ohwoviorole AE, Parkes AB, Lazarus JH. Prevalence Of thyroid autoantibodies in Nigeria Patients. QJM. 2007;100(2):107-12.

28. Gunasekara P, Hettiarachchi M, Liyanage C, Lekamwasam S. Effects of zinc and multimineral vitamin supplementation on glycaemic and lipid control in adult diabetes. Diabetes Metab Syndr Obes. 2011;4:53-60.

29. Lang TJ. Estrogen as an immunomodulatory. Clin Immunol. 2004;113:224-30.

30. Fairweather D, Frisando-Kiss S, Rose NR. Sex differences in Autoimmune diseases from pathological perspective. Am J Pathol. 2008;173(3):600-9.

31. Pearce EN, Farwell AP, Braverman LE. Thyroiditis. N Eng J Med. 2003;348:2646.

32. Trbojević B, Djurica S. Diagnosis of autoimmune thyroid disease. Srp Arh Celok Lek. 2005;133 Suppl 1:25-33.

33. Spencer CA. Challenges of serum thyroglobulin $(\mathrm{Tg})$ measurement in the presence of Tg autoantibodies. J Clin Endocrinol Metab. 2004;89:3702-4.

34. Chubb SAP, Davis WA, Inman Z, Davis TME. Prevalence and progression of subclinical hypothyroidism in women with type 2 diabetes: The Fremantle Diabetes Study. Clin Endocrinol. 2005;62:480-6.

35. Hage M, Zantout MSM, Azar ST. Thyroid disorder and diabetes mellitus. J Thyroid Res. 2011;2011:439463.

36. Chehade JM, Gladysz M, Mooradian AD. Dyslipidaemia in type 2 diabetes: Prevalence, pathophysiology, and management. Drugs. 2013;73(4):327-39.

37. Primo ME, Niepomniszcze H, Poskus E, Sala MS, Guaita SS, Sica MP, Villalba A, Cardoso A, Bruno OD. Frequency of pancreatic beta-cell autoimmunity markers in patients with autoimmune thyroid disease. Medicina (B Aires). 2008:68(1):37-42.

\section{Submit your next manuscript to BioMed Central and we will help you at every step:}

- We accept pre-submission inquiries

- Our selector tool helps you to find the most relevant journal

- We provide round the clock customer support

- Convenient online submission

- Thorough peer review

- Inclusion in PubMed and all major indexing services

- Maximum visibility for your research

Submit your manuscript at www.biomedcentral.com/submit

) Biomed Central 